\section{One size doesn't fit all}

In his Comment article in the May issue of Microbiology (Ferenci, 2006), Dr Ferenci provides a very timely 'pull on the reigns' and reminds us that what we all have been taught classically about continuous cultures is a gross oversimplification. Pragmatically, or by necessity if you like, the theory of the continuous culture is based upon a simple mathematical model. If we look at the constants in that model, $\mu_{\max }$ and $K_{\mathrm{s}}$, we really should stop to think. These are not invariable within a population of cells and, as such, mutations will sweep through a population due to selective pressures (as indicated by Dr Ferenci). These mutant 'take-overs' have been described in several studies, and have been shown to be strain-dependent. Indeed, our laboratories work mainly with Streptomyces coelicolor, where visually obvious mutants (asporulent and pigmented antibiotic variants) occur frequently upon prolonged continuous culture. These strain variabilities and mutational effects are approached in our laboratories by restricting prolonged studies (reduction in generation times) when the mutational aspect is not the reason for study, thus material required for analysis is harvested on or at the establishment of (quasi-) steady-state, especially for global studies. With respect to our previous article (Hoskisson \& Hobbs, 2005), we failed to indicate that the establishment of (quasi-) steady-state under empirically established conditions may be problematic in the initiation of studies, and a careful experimental design strategy is required.

Mutations aside, one should also carefully consider $K_{\mathrm{s}}$, the substrate affinity constant. Substrate uptake mechanisms are often composed of multiple transporters for nutrients. These nutrients can often have carrier molecules facilitating their 'capture' and binding (e.g. siderophores involved in ion binding). These transporters and siderophores are not mutually exclusive and considering the term $K_{\mathrm{s}}$ as a 'single' constant is probably the greatest fault in the mathematical model. Monod's approach of producing a simple mathematical model remains valid today. However, it should be tempered by an appreciation of the fact that what we are dealing with are highly complex biological systems.

It is not doubted that continuous culture systems are of huge benefit to a multitude of studies, and the study of bacterial evolution and selection processes are a major beneficiary of the versatility of continuous culture. However, I feel that, providing the pitfalls outlined by Dr Ferenci are appreciated, the initiation of global studies of transcription, proteomics and metabolomics in continuous culture systems will provide useful insights into the biology of bacterial physiology.

\section{P. A. Hoskisson ${ }^{1}$ and G. Hobbs ${ }^{2}$}

${ }^{1}$ Department of Molecular and Cell Biology, University of Aberdeen, Institute of Medical Science, Foresterhill, Aberdeen AB25 2ZD, UK

${ }^{2}$ School of Biomolecular Sciences, Liverpool John Moores University, Byrom Street, Liverpool L3 3AF, UK

Correspondence: G. Hobbs (g.hobbs@livjm.ac.uk)

Ferenci, T. (2006). A cultural divide on the use of chemostats. Microbiology 152, 1247-1248.

Hoskisson, P. A. \& Hobbs, G. (2005).

Continuous culture - making a comeback? Microbiology 151, 3153-3159.

\section{DOI 10.1099/mic.0.29073-0}

\section{On the enhancement of anti-neurotoxin antibody production by subcomponents HA1 and HA3b of Clostridium botulinum type B 165 toxin-haemagglutinin}

In a recent article in this journal, Lee et al. (2005) reported that immunization of mice with formaldehyde-treated botulinum neurotoxin B (BoNT/B) in the presence of haemagglutinin subcomponents HA1 and $\mathrm{HA} 3 \mathrm{~b}$, normally present in the $16 \mathrm{~S}$ toxin, produced higher levels of anti-BoNT/B antibodies (Abs) than immunization with BoNT/B alone. Because of the paper's potential clinical therapeutic implications, this comment discusses three major issues in the paper: quality of the antigen, dose and frequency of immunization. I show that these were quite different from those used in therapeutic applications of active BoNT/B (or any BoNT) complex.

BoNTs (mostly types A and B) are used to treat a variety of clinical conditions associated with involuntary muscle spasm and contractions as well as in cosmetic and other therapeutic applications (Jankovic, 2002, 2004; Atassi \& Oshima, 1999; Brashear et al., 2004; Dressler \& Bigalke, 2005). The therapeutic benefits are of limited duration and toxin injections need to be repeated every 3-6 months. In a very small percentage of patients (less with toxin A than with toxin B) the treatment elicits blocking $\mathrm{Ab}$ responses against the correlate toxin, which reduce or completely terminate the patient's responsiveness to further treatment (Göschel et al., 1997; Atassi \& Oshima, 1999; Atassi, 2002, 2004;

Microbiology Comment provides a forum for discussion of scientific issues arising directly from papers that have been published in the pages of Microbiology. The authors of papers under discussion will be offered an opportunity to respond.

Guidelines on how to submit a Microbiology Comment article can be found in the Instructions for Authors at http://mic.sgmjournals.org

It should be noted that the Editors of Microbiology do not necessarily agree with the views expressed in Microbiology Comment.

Charles J. Dorman, Editor-in-Chief 